\title{
Griffiths phases on complex networks
}

\author{
Miguel A. Muñoz, ${ }^{1}$ Róbert Juhász, ${ }^{2}$ Claudio Castellano, ${ }^{3}$ and Géza Ódor ${ }^{4}$ \\ ${ }^{1}$ Institute Carlos I for Theoretical and Comp. Physics. Universidad de Granada, 18071 Granada, Spain. \\ ${ }^{2}$ Research Institute for Solid State Physics and Optics, H-1525 Budapest, P.O.Box 49, Hungary \\ ${ }^{3}$ CNR-ISC, Unità SMC and Dip. di Fisica, Univ. di Roma "La Sapienza", P. A. Moro 2, 00185 Roma, Italy \\ ${ }^{4}$ Research Institute for Technical Physics and Materials Science, H-1525 Budapest, P.O.Box 49, Hungary
}

\begin{abstract}
Quenched disorder is known to play a relevant role in dynamical processes and phase transitions. Its effects on the dynamics of complex networks have hardly been studied. Aimed at filling this gap, we analyze the Contact Process, i.e. the simplest propagation model, with quenched disorder on complex networks. We find Griffiths phases and other rare region effects, leading rather generically to anomalously slow (algebraic, logarithmic, ...) relaxation, on Erdős-Rényi networks. Similar effects are predicted to exist for other topologies with a finite percolation threshold. More surprisingly, we find that Griffiths phases can also emerge in the absence of quenched disorder, as a consequence of topological heterogeneity in networks with finite topological dimension. These results have a broad spectrum of implications for propagation phenomena and other dynamical processes on networks.
\end{abstract}

PACS numbers: 89.75.Fb, 89.75.-k, 05.90.+m

Networks have become a paradigm in the study of complex systems [1]. After initial efforts devoted to uncover their non-trivial topological features, the focus shifted to dynamical processes occurring on them [2]. Models of epidemics [3], such as the susceptible -infected -recovered (SIR), the susceptible -infected -susceptible (SIS), or the contact process (CP), have played a prominent role [2]. The concept of "epidemics" covers a broad variety of processes including real epidemics, computer viruses, rumor spreading, or signal propagation in neural nets. A remarkable finding is the absence of a finite infection threshold for the SIS process in heterogeneous scale-free networks; i.e. in contrast with regular lattices, where a critical point separates an active from an absorbing phase 3], these networks host endemic states even for arbitrarily small infection rates [2]; i.e. topological disorder crucially affects dynamical processes on networks.

Quenched disorder is well known to induce novel behavior (such as, universality changes) in phase transitions both in equilibrium and away from it. Under some circumstances, it may also generate phases unheard-of in pure systems. Consider, for illustration, the quenched contact process (QCP) [4], characterized by a site-dependent quenched infection rate, on a lattice. Even if the system is globally in its absorbing phase, disorder fluctuations can generate rare active regions with over-average infection rate. In these, activity lingers for extremely long periods; however, being finite, they ineluctably end up falling into the absorbing state. The convolution of exponentially rare regions with exponentially large surviving times gives rise (see below) to a region in the absorbing phase, characterized by a generic algebraic decay of activity, i.e. a Griffiths phase (GP) [4] 6 .

This is just an example of a generic phenomenon, thoroughly studied in the disordered systems literature in classical, quantum, and non-equilibrium systems [5], oc- curring whenever exponentially distributed cluster sizes, $P(s) \sim \exp (-c s)$, have exponentially long activity times, $\tau(s) \sim \exp (b s)$; this leads to a GP with algebraic decay $\rho \sim \tau^{-c / b}$ with a continuously varying exponent (see [5] for analytical approaches, further details and applications, and [6] for a recent review). Observe that, on the opposite to usual critical points, in GPs one observes scale-invariance generically, i.e. without the need of parameter fine-tuning. Similarly, other size distributions (as power-laws) do lead to different functional forms of slow relaxation. Let us stress that many disorder effects appear rather similarly in equilibrium and away from it; for instance, the (strong disorder) critical point of the (non-equilibrium) QCP is in the same universality class as the (equilibrium) random transverse-field Ising model [7]; i.e. rare-region effects transcend the frontier between equilibrium and non-equilibrium.

From this broad perspective, it is surprising that very little attention has been paid so far to the effect of quenched disorder on dynamical processes on complex networks. Heterogeneity in the intrinsic properties of nodes is a very natural (not to say, unavoidable) feature of real networks: node-dependent rates appear in all the examples above, owing to the specificity of the individual immune response, presence of antivirus software, and so forth. Our aim here is twofold: (i) we tackle the study of quenched node disorder in dynamical processes on complex networks, and look for rareregion effects in the simplest possible epidemic model, i.e. the QCP on Erdös-Rényi (ER) random networks; (ii) we explore whether network topological disorder on its own can induce rare-region effects. We report on the existence of Griffiths effects, including various non-trivial regimes with generic slow decay of activity, for different networks with quenched node disorder and/or topological disorder. Our main conclusion is that disorder, either quenched or topological, may induce under the conditions 
studied here, slow relaxation on network dynamics; this is expected to go beyond the considered examples, and to apply to different models, dynamics and topologies.

In the pure Contact Process, infected/active nodes heal at rate $\mu$ and infect a randomly chosen nearest-neighbor (provided it was un-infected) at rate $\lambda$. A simple (onesite) mean-field treatment of the average activity density, $\rho$, predicts an absorbing phase transition at $\lambda_{c}^{1}=\mu$, i.e. where the infection and healing rate compensate each other [8]. This prediction for the threshold value is not correct for finitely connected networks since in that case activity appears in localized regions, reducing the effective infection rate (i.e. the prob. of having an already infected neighbor is larger than in the perfect mean-field mixing), i.e. $\lambda_{c}>\lambda_{c}^{1}$. A lengthy but standard [3] two-site or "pair" approximation, leads to $\lambda_{c}^{2}=\mu\langle k\rangle /(\langle k\rangle-1)$, where $\langle k\rangle$ is the average network degree [9]. Observe that the correction factor with respect to $\lambda_{c}^{1}$ converges to 1 when $\langle k\rangle \rightarrow \infty$ (i.e. where mean-field holds) and diverges at the percolation threshold $\langle k\rangle=1$ below which the network is fragmented [2, 10], it cannot sustain activity and the phase transition disappears.

We consider a $Q C P$ [4] with $\mu=1$ and a quenched disordered infection rate: a fraction $1-q$ of the nodes (type-I) take value $\lambda$ and the remaining fraction $q$ (typeII nodes) take a reduced value $\lambda r$, with $0 \leq r<1$. Obviously, for $q=0$ and $q=1$ the model is pure, $\lambda_{c}(q=1)=\lambda_{c}(q=0) / r$, while for $0<q<1$ one expects $\lambda_{c}$ to interpolate between these limits. For simplicity, we fix $r=0$ from now on. The average activity density is $\rho=(1-q) \rho_{1}+q \rho_{2}$ (subscripts standing for node type) and the one-site mean-field equations are $\dot{\rho}_{i}(t)=-\rho_{i}+\lambda\left(1-\rho_{1}-\rho_{2}\right)\left((1-q) \rho_{1}\right)$ for $i=1,2$, whose stationary solution has a critical point at $\lambda_{c}^{1}(q)=1 /(1-q)$. As above, the one-site result needs to be corrected by a factor $\langle k\rangle /(\langle k\rangle-1)$ to account for activity clustering, leading to:

$$
\lambda_{c}^{2}(q)=\frac{\langle k\rangle}{\langle k\rangle-1} \frac{1}{1-q} .
$$

Relevant for what follows is that type-I nodes experience a percolation transition where the type I-to-type I average degree is 1 [2, 10], i.e. at $q_{\text {perc }}=1-\langle k\rangle^{-1}$. For $q>q_{\text {perc }}$ activity cannot be sustained: type-I clusters are finite and type-II ones do not propagate activity.

We now present the results of a numerical investigation of the QCP on ER networks with $\langle k\rangle=3$ (implying $q_{\text {perc }}=2 / 3$ ), and sizes up to $N=10^{7}$. Simulations are performed in a standard way: all sites are declared active initially and the dynamics proceeds as follows [3]. A site, $i$, is randomly selected and it either heals (with prob. $1 /\left(1+\lambda_{i}\right)$ ) or infects a randomly selected neighbor provided it was empty (with prob. $\lambda_{i} /\left(1+\lambda_{i}\right)$, where $\lambda_{i}=\lambda$ or 0 depending on site type). We monitor the activity decay averaged over
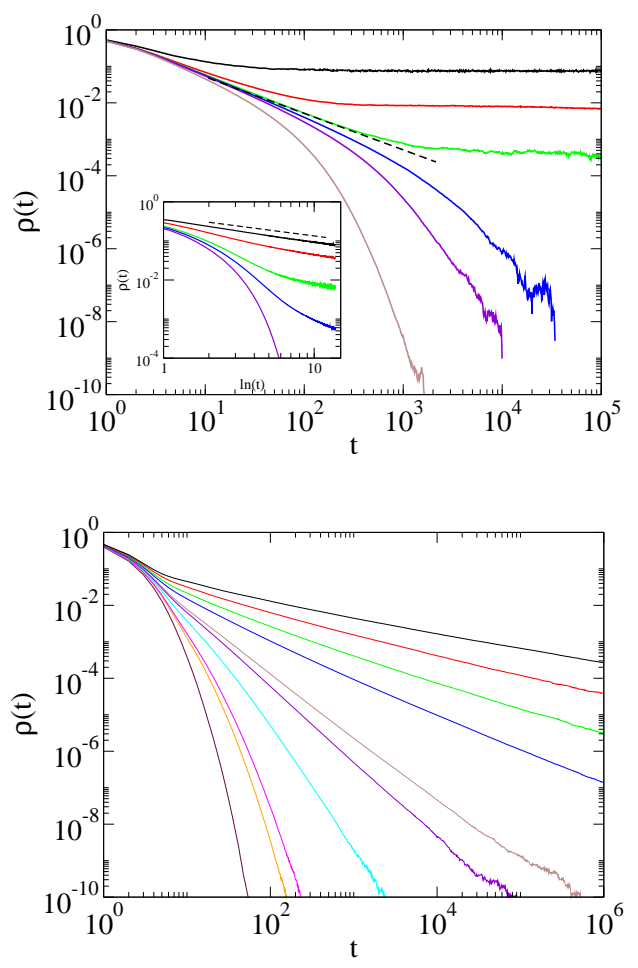

FIG. 1: Average activity density $\rho(t)$ vs time $t$ for ER networks with $\langle k\rangle=3, r=0$, and $N=10^{5}$. $\lambda$ s are ordered from top to bottom in all panels. (a) Upper panel: $q=0.6$, and $\lambda=5,3.8,3.6,3.55,3.5,3.3$; the dashed line is proportional to $t^{-1}$. (a) Inset: $\rho$ vs $\ln (t)$ for $q=2 / 3 ; \lambda=10,7,5,4.5$, 4 ; the dashed line is proportional to $\ln (t)^{-1 / 2}$. (b) Lower panel: $q=0.9$, and $\lambda=50,30,20,15,10,9,7,5,4.5,2.7$. Straight lines lie in the Griffiths phase.

many runs. Numerical results are synthesized in Fig. 1 and the inferred phase-diagram is summarized in Fig. 2. For $q<2 / 3$ a critical line $\lambda_{c}(q)$, which is very well fitted by Eq. (11) (implying that the pair approximation captures the most relevant correlations), separates an active phase from an absorbing one (Fig. 1 $\mathrm{a}$ ). Instead for the fragmented case, $q>2 / 3$ there is no active phase, as predicted above (Fig. 1b). The absorbing phase can be divided into various sub-phases. For $q>2 / 3$ and $\lambda(q)>\lambda_{c}\left(q=q_{\text {perc }}\right) \approx 4.5$ we observe (Fig. 1b) a powerlaw decay with continuously varying exponents: $\rho(t) \sim t^{-\theta(q, \lambda)}$, with $\theta \rightarrow 0 q \rightarrow q=2 / 3$ i.e. a GP. Right at $q=2 / 3$ we find a logarithmic decay $\rho(t) \sim \ln (t)^{-1 / 2}$ for $\lambda>\lambda_{c}\left(q_{\text {perc }}\right) \approx 4.5$ (Fig. 19 (inset)). For $q>2 / 3$ and $\lambda_{c}(q=0)<\lambda<\lambda_{c}\left(q_{\text {perc }}\right)$, as well as for $q<2 / 3$ and $\lambda_{c}(q=0)<\lambda<\lambda_{c}(q)$ (see Fig. 2), we can fit a stretched exponential (not shown). Finally, below the threshold of the pure system $\lambda_{c}(q=0) \approx 1.5$ the decay is purely exponential.

Let us now rationalize these observations by using, as customarily done in disordered systems (see [4 $[6,11]$ ), optimal fluctuation arguments. The following regimes can be predicted: (i) Griffiths phase: $\lambda>\lambda_{c}\left(q_{\text {perc }}\right)$ and 


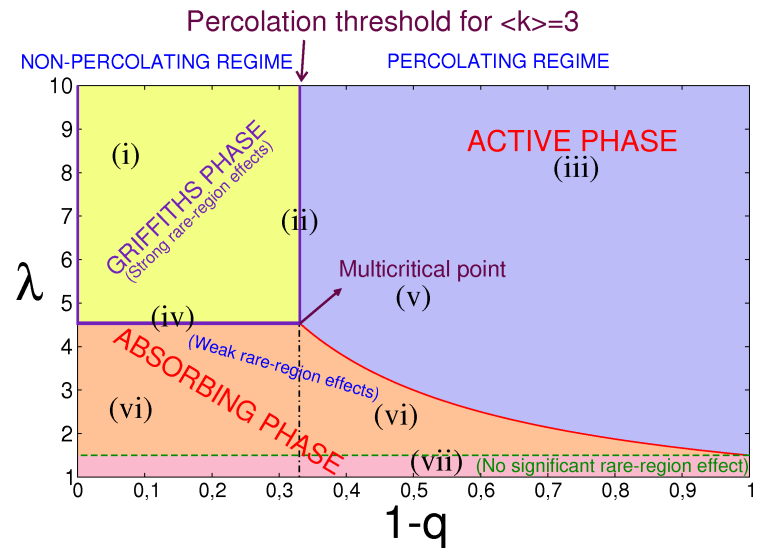

FIG. 2: Phase diagram for $r=0$. See main text for details.

$q>q_{\text {perc }}$. The cluster size distribution for ER networks in the fragmented case is [12]:

$$
P(s) \sim \frac{1}{\sqrt{2 \pi} p} s^{-3 / 2} e^{-s(p-1-\ln (p))}
$$

where $s$ is the cluster size and $p$ is the average number of links per node, which in our case is $p=\langle k\rangle_{q=0}(1-q)$ for type-I nodes. Within any given cluster of typeI nodes, let us define $p_{l o c}$ as the local average number of links per node; and from it, a local value of $q, q_{l o c}=1-p_{l o c} /\langle k\rangle_{q=0}$. Obviously, for connected type-I clusters are above the percolation threshold, i.e. $q_{\text {loc }}<q_{\text {perc }}$, and hence, if $\lambda>\lambda_{c}\left(q_{\text {perc }}\right)$, they are active rare regions where activity survives until a coherent fluctuation extinguishes it. This occurs after a characteristic time $\tau(s)$ which grows exponentially with cluster size, i.e. $\tau \simeq t_{0} \exp (A(\lambda) s)$, where $t_{0}$ and $A(\lambda)$ do not depend on $s$. Hence, the overall activity decays as:

$$
\rho(t) \sim \int d s \text { s } P(s) \exp \left[-t /\left(t_{0} e^{A(\lambda) s}\right)\right] .
$$

Plugging Eq. (2) into Eq. (3) and using a saddle point approximation, one obtains $\rho(t) \sim t^{-\theta(p, \lambda)}$, with $\theta(p, \lambda)=$ $-(p-1-\ln (p)) / A(\lambda)$; i.e. there is a generic power-law decay with continuously varying exponents, i.e. a GP. Note that $\theta \rightarrow 0$ when $p \rightarrow 1$, suggesting a crossover to logarithmic decay. (ii) Indeed, right at the percolation threshold, $q=q_{\text {perc }}$, the cluster size distribution, Eq. (2), becomes a power-law leading, when plugged in Eq. (3), to a logarithmic decay, $\rho(t) \sim\left[\ln \left(t / t_{0}\right)\right]^{-1 / 2}$, for any $\lambda$ larger than $\lambda_{c}\left(q_{\text {perc }}\right)$ (for which rare regions are active). (iii) For $q<q_{\text {perc }}$ there is a giant component of typeI nodes and, hence, standard mean-field like contactprocess behavior is expected; i.e. power-law decay, $\rho(t) \sim t^{-1}$ at criticality. Similar arguments lead to: (iv) In the absorbing region with $\lambda_{c}(q=0)<\lambda<\lambda_{c}\left(q_{p e r c}\right)$, rare regions with $q_{l o c}<q_{\text {perc }}$ exist but, in contrast to previous cases, they are subcritical (activity decays exponentially fast in each of them); a stretched exponential fits the decay. Active rare clusters can also appear; but they require $q_{l o c}$ to be smaller that the critical value of $q$ for the given $\lambda$, a stringent condition, leading (in all the region (iv) in Fig.(2)) to weak rare-region effects (as opposed to the previous strong effects) [9]. Finally, region (v) with $\lambda<\lambda_{c}(q=0)$ is free from rare-region effects: all clusters are subcritical with exponential decay.

Summing up, optimal fluctuation arguments explain all numerical findings. Rare regions play a key role giving rise to generic slow decay of activity. Similar results hold for any value of $r$, i.e. when activity flows out of any site. Hence, strong rare-region effects are expected to occur in generic networks with a finite percolation threshold (e.g. structured scale-free networks [13] or networks with communities), while if the percolation threshold vanishes (e.g. Barabási-Albert scale-free nets [1]) only weak rareregion effects are predicted.

In what follows, we investigate whether GPs induced merely by topological disorder can exist. Let us first recall that Eq.(11) provides an excellent approximation to the $(\langle k\rangle$-dependent) critical point. As a consequence, networks with heterogeneous degree may, in principle, exhibit topological rare regions: clusters with local degree, $k$, above average would have a smaller percolation threshold and could be locally active even if the whole network is absorbing. For argument's sake, let us consider the CP on a network with bimodal degree distribution, $P(k)=p \delta\left(k-k_{1}\right)+(1-p) \delta\left(k-k_{2}\right)$ with $k_{1} \gg k_{2}$; a priori, one could expect rare active regions (with overdensity of $k_{1}$-nodes) to exist. However, numerically we find just conventional, non-disordered, exponential decay. Why is it so? In $d$-dimensional lattices disorder is known to be irrelevant for large $d$; the number of nearest neighbors is so high that the central limit theorem precludes rare regions (i.e. deviations from normality) from existing. An extension of the concept of Euclidean dimension to arbitrary graphs is the topological dimension, $D$, which measures how the number of nodes in a neighborhood grows as a function of the topological distance from an arbitrary origin: $N(l) \sim l^{D}[10]$. In parallel with the result for lattices, we conjecture that GPs and similar rare-region effects do not typically exist for the $\mathrm{CP}$ in networks with an infinite topological dimension (such as ER graphs above their percolation threshold or the discussed bimodal graphs), justifying our numerical findings. Instead, for ER below the percolation threshold, which are characterized by a vanishing effective topological dimension, $D=0$ (i.e. the number of nodes in any neighborhood/cluster converges to a constant for large values of $l$ ) GPs can exist. This suggests to explore CP on networks with finite $D$. As an example, we take the generalized random small-world networks defined in [14] as follows. Starting with a ring of $L$ nodes, one defines the distance between nodes $i$ and $j$ as $l=\min (|i-j|, L-|i-j|)$; all nearest neighbors are connected and any pair with $l>1$ is connected with probability $P(l)=1-\exp \left(-\beta l^{-\alpha}\right)$. We have identified three different cases: i) For $\alpha<2$ 


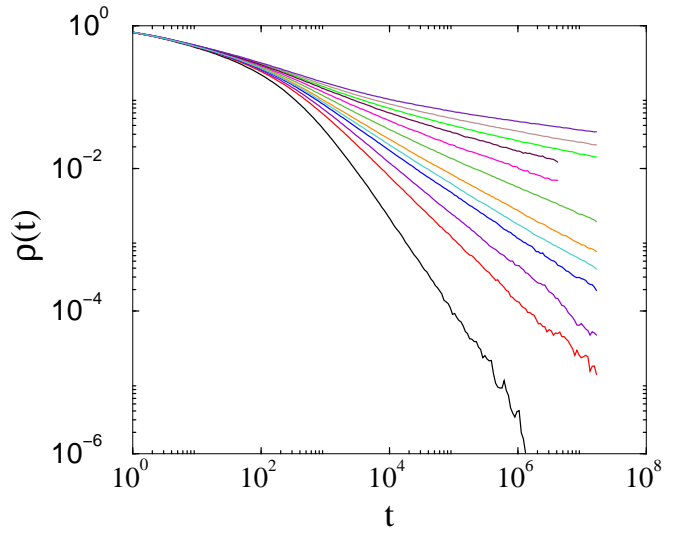

FIG. 3: Density decay in generalized small-world networks with $\alpha=2$ and $\beta=0.2$ for different values of $\lambda$ (from top to bottom: 2.81, 2.795, 2.782, 2.77, 2.75, 2.73, 2.71, 2.70, $2.69,2.67,2.65,2.6)$. Straight lines lie in the Griffiths phase.

the network diameter grows poly-logarithmically with $N$, hence, formally $D=\infty$; indeed, as conjectured, no Griffiths phases are observed in our numerics (not shown). The critical behavior seems to correspond to that of the contact process with Lévy-flight jumps [3]. ii) For $\alpha>2,\langle l\rangle$ is finite, and correspondingly $D=1$; longedges do not alter the system dimensionality but they do introduce quenched disorder and a GP. We conjecture the critical behavior to coincide with that of the one-dimensional infinite randomness fixed point of the quenched contact process [6, 7], as indeed verified numerically (not shown); topological and quenched disorder can lead to the same universal behavior. iii) In the marginal case $\alpha=2, D$ has been conjectured to be $\beta$-dependent, $D=D(\beta)[14,15]$. We have checked that $D$ is indeed an increasing function of $\beta(D(0)=1, D(0.2) \approx 1.21$, $D(0.5) \approx 2.3)$. We expect the critical point $\lambda_{c}(\beta)$ to decrease when $\beta$ grows (i.e. when $\langle k\rangle$ increases; see Eq.(11)). Hence, for a given $\beta$, the model must be in the active phase if $\lambda>\lambda_{c}(0)=3.297848(22)$ (critical point in the one-dimensional lattice [3]) and must be inactive if $\lambda<\lim _{\beta \rightarrow \infty} \lambda_{c}(\beta)=1$ (critical point for the fully connected graph). Therefore, $\lambda_{c}(\infty) \leq \lambda_{c}(\beta) \leq \lambda_{c}(0)$ and the possible GP is bounded by this interval. Numerical simulations for $\beta=0.2$ confirm (Fig. 3) the existence of a GP with generic power-law decay for $\lambda$ in $[2.65,2.81]$. The width of the GP decreases with increasing $\beta$ (i.e. the larger $D$ the smaller the rare region effects); actually, preliminary results, suggests the existence of a finite upper critical dimension above which the GP disappears [9]. We have found similar topological GPs for other smallworld nets, and we predict them to emerge in many other networks, such as spatially embedded networks or fractal [16] scale-free ones, with finite topological dimension.

In summary, taking the contact process as an example we have shown that quenched disorder can induce GPs and other rare-region effects leading to generic slow relaxation for dynamical processes on ER networks. Similar effects are argued to appear on other topologies with a finite percolation threshold. Furthermore, heterogeneity in the topology may suffice to generate GPs on its own. This can occur only if the network topological dimension is not infinite allowing for rare regions to exist. Our results are expected to apply to dynamical processes other than the CP (even if each case should be carefully examined). An inspiring application, illustrating Griffiths effects at play, is provided by a recent work reporting on algebraic "forgetting" times (reproducing experimental findings) in a simple model of memory [17]; this generic slow decay crucially depends on the neural network topological disorder. Social networks with heterogeneous communities have also reported to exhibit generic slow decay and, what we interpret as, severe rare-region effects [18].

This work was supported by HPC-EUROPA2 pr.228398, HUNGRID and Hungarian OTKA (T77629,K75324), J. de Andalucía P09-FQM4682 and MICINN-FEDER project FIS2009-08451.

[1] R. Albert, A.L. Barabási, Rev. Mod. Phys. 74, 47 (2002).

[2] S. N. Dorogovtsev, A. V. Goltsev, J. F. F. Mendes, Rev. Mod. Phys. 80, 1275 (2008).

[3] J. Marro and R. Dickman, Non-equilibrium phase transitions in lattice models, Cambridge Univ. Press, (Cambridge 2005). G. Ódor, Rev. Mod. Phys. 76, 663 (2004).

[4] A. J. Noest, Phys. Rev. Lett. 57, 91 (1986).

[5] R. B. Griffiths, Phys. Rev. Lett. 23, 17 (1969). A. J. Bray, Phys. Rev. Lett. 59, 586 (1987). D. Dhar, M. Randeira, J. P. Sethna, Europhys. Lett. 5, 485 (1988). H. Rieger and A. P. Young, Phys. Rev. B 54, 3328 (1996). D.S. Fisher, Phys. Rev. Lett. 69, 534 (1992).

[6] T. Vojta, J. Phys. A: Math. Gen. 39, R143 (2006).

[7] J. Hooyberghs, F. Iglói, and C. Vanderzande, Phys. Rev. Lett. 90, 100601 (2003).

[8] C. Castellano and R. Pastor-Satorras, Phys. Rev. Lett. 96, 038701 (2006).

[9] C. Castellano, G. Ódor, R. Juhász, and M.A. Muñoz, Preprint 2010.

[10] B. Bollobás, Random Graphs, Cambridge Studies in Adv. Math. 73. Cambridge University Press, Cambridge, 2001.

[11] M.Y. Lee and T. Vojta, Phys. Rev. E 79, 041112 (2009).

[12] A. J. Bray and G. J. Rodgers, Phys. Rev. B 38, 11461 (1988).

[13] V. M. Eguíluz and K. Klemm, Phys. Rev. Lett. 89, 108701 (2002).

[14] I. Benjamini and N. Berger, Rand. Struct. Alg. 19, 102 (2001).

[15] D. Coppersmith, D. Gamarnik, and M. Sviridenko, Rand. Struct. Alg. 21, 1 (2002).

[16] C. Song, S. Havlin, and H.A. Makse, Nature 433, 392 (2005).

[17] S. Johnson, J. J. Torres, and J. Marro, arXiv:1007.3122

[18] X. Castelló, et al., EPL, 79, 66006 (2007). R. Toivonen et al. Phys. Rev. E 79, 016109 (2009). P.J. Mucha et al, Science 328, 876 (2010). Karsai et al. arXiv:1006.2125 [physics.soc-ph] 